\title{
Alcohol Related Birth Defect
}

National Cancer Institute

\section{Source}

National Cancer Institute. Alcohol Related Birth Defect. NCI Thesaurus. Code 692727.

A physical or cog nitive mental abnormality caused by maternal alcohol consumption and its toxic effect on the developing embryo during pregnancy. 